\title{
Validation and Evaluation of COSINE Software Package Based on the Operation Data of Third Generation Passive Pressurized Water Reactor
}

\author{
Weibin $\mathrm{ZHANG}^{\mathrm{a}, 1}$, Chenglin $\mathrm{ZHU}^{\mathrm{b}, \mathrm{c}}$, Qiao ZHANG ${ }^{\mathrm{a}}$, Linlin $\mathrm{XU}^{\mathrm{a}}$ and Guoping \\ QUAN $^{\mathrm{b}, \mathrm{c}}$ \\ a Shandong Nuclear Power Co., Ltd, Yantan 265116, Shandong, China \\ ${ }^{\mathrm{b}}$ National Electric Energy Investment Group Scientific and Technology Co., Ltd, \\ Beijing 102209, China \\ ${ }^{c}$ National Energy Key Laboratory of Nuclear Power Software, Beijing 102209, China
}

\begin{abstract}
According to the historical experience of international nuclear power software development and the requirements of relevant guidelines at home and abroad, a large number of experiments and theoretical work must be carried out to verify and confirm the empirical formulas, models and calculation methods used in the software and evaluate the models related to safety evaluation in order to make the software be applied to the design and analysis of nuclear power plants. Validation and evaluation is the most important key link in the process of nuclear power software development, which is heavy workload and difficult, and needs a lot of actual power plant operation data. This paper proposed a research on the validation and evaluation of the COSINE software package's calculation capability and accuracy based on the operation data of the third generation passive PWR (Pressurized Water Reactor) AP1000. The comparison results between the operation limit parameters of the nuclear power plant including critical boron concentration, heat pipe factor of nuclear enthalpy rise, heat flux hot spot factor and AO (Axial Offset) showed that the data calculated by COSINE met the running requirements of the nuclear power plant, and the calculation accuracy keeps also in a good way.
\end{abstract}

Keywords. COSINE, passive PWR, validation and evaluation

\section{Introduction}

Started up from August 2018, Units 1 and 2 of the Haiyang Nuclear Power Plant has finished the refueling and overhauling for the first time until May 2020. And the operation data of the two units has been saved during the whole running time. At the same time, in the verification and validation process of the COSINE [1-2] software package, this paper proposed a research on the evaluation of COSINE's calculation capability and accuracy cooperating with the calculation verification of the software by National Electric Energy Investment Group Central Institute.

1 Corresponding Author, Weibin ZHANG, Shandong Nuclear Power Co., Ltd, Yantan 265116, Shandong, China; Email: 934071441@qq.com. 
COSINE (COre and System INtegrated Engine for design and analysis) is a kind of nuclear design integration package on safety analysis and engineering design for nuclear power plant. It is an important and special project projected by National Energy Administration and built by National Electric Energy Investment Group Scientific and Technology Co., Ltd, Beijing Software Technology Center. This project aims at developing China's first independent intellectual property capable for the design and safety analysis of the second and third generations of passive PWRs. Included in the COSINE software package, cosNu, a design software of reactor core, contains of the parameter calculation component cosLATC [3] and core physics analysis component cosCORE [4].

During the development process of the software, the program development and verification work is done firstly, including requirements analysis, program design, code writing, program debugging, program verification, etc. And then a draft software is developed, verified, and evaluated by developing an evaluation model. Validation and evaluation is a core process during the development which is with a huge workload, a high difficulty and a massive amount of operation data from various types of power plants.

This paper proposed a research on the validation and evaluation of the COSINE software package's calculation capability and accuracy based on the real operation data of the third generation passive PWR (Pressurized Water Reactor) AP1000.

\section{Brief Introduction of Haiyang Nuclear Power Plant}

Units 1 and 2 of Haiyang Nuclear Power Plant are AP1000 reactor, which is a two-loop passive pressurized light water reactor with 157 fuel assemblies of AP1000, rated output power of $3400 \mathrm{MW}$ and active zone height of $426.72 \mathrm{~cm}$. Each of the assembly consists of 264 fuel rods, 24 control rod guide tubes and a neutron fluence rate measuring tube arranged as $17 \times 17$ square grid. And, each of the assembly has $8 \mathrm{mid}$ span spacer grids, 4 mid span mixing grids, a top grid, a bottom grid and a protection grid. Mid span spacer grids and mixing grids are all alloy ZIRLOTM while top grid, bottom grid and protection grid are all INCONEL.

Five zones of A, B, C, D and E are arranged in the way of advanced low-leakage loading pattern [5] in the first cycle reactor core and each of the zone is with different average enrichments (axial regeneration zone is not included) of $0.74 \%, 1.58 \%, 3.05 \%$, $3.72 \%$ and $4.27 \%$ respectively. And at the same time, the numbers of fuel assemblies are 16, 49, 28, 36 and 28 correspondingly. Burnable assemblies are Integer fuel burnable assembly (IFBA) and wet annular burnable assembly (WABA). And 5632 IFBAs and 592 WABAs are used in the first cycle to control part of the excess reactivity in the reactor core.

There are 69 rod cluster control assemblies in total, in which 53 are RCCA (Rod Cluster Control Assembly) with alloys Ag-In-Cd absorber and 16 are GRCA (Gray Cluster Control Assembly) with tungsten absorber.

\section{Validation and Evaluation Methods}

Applied in the reactor core of Haiyang Nuclear Power Plant, the FID (Fixed In-core Detector) [6-7] can transmit the current signal in it to the reactor core monitoring 
software in real time. With the parameters of the power of the plant, the position of the control rod and the average temperature as the real-time power distribution indication for the plant, the operation limit parameters of the reactor can be calculated and saved every $15 \mathrm{~min}$. And this is the data basis for the software verification. Then according to the sampling time of boron concentration, the corresponding reactor core typical operation limit parameters are selected as the data points. To select effective points, points with low or fluctuated power, and failed to reach xenon equilibrium condition are removed. Besides, a certain fuel consumption interval is applied to extract the data within the requirements. Finally, 43 state points of Unit 1 and 44 state points of Unit 2 are chosen for the validation and evaluation of COSINE.

Included in the COSINE software package, $\cos \mathrm{Nu}$, a design software of reactor core, is applied to the modelling work of Haiyang Nuclear Power Plant. There are two parts in the modelling work: firstly, cosLATC is used for the core assemblies modelling to calculate and generate a few groups cross section database; and cosCORE is used for core modelling to calculate the operation conditions based on the database. Due to the fact that cosLATC is a two-dimensional computer program, it separates the assemblies into 15 kinds according to enrichment, IFBA, WABA and axial layout.

Reactor core modelling consists of two parts: core parameter information and function calculation; AP1000 reactor core parameters modelling consist of core thermal parameters, geometric parameters, material parameters, reflector parameters, control rod parameters, grid parameters, etc. In regard to the contents of the core operation parameters, a model is built for the validation and evaluation based on the burnup interval of the selected points of Units 1 and 2 by calculating the critical boron concentration, heat pipe factor of nuclear enthalpy rise, heat flux hot spot factor and AO (Axial Offset). The calculated and real operation results are compared and analyzed, and the calculation accuracy is also evaluated.

\section{Results}

The first cycle operation data of Units 1 and 2 of Haiyang Nuclear Power Plant are used to evaluate the COSINE's calculation results on the critical boron concentration, heat pipe factor of nuclear enthalpy rise, heat flux hot spot factor and AO at full power in this paper. And the evaluation is made from the point of view of power plant operation.

\subsection{Critical Boron Concentration}

The deviation of critical boron concentration between the observed value in site and the value calculated by cosNU is shown in figure 1 . The acceptance criteria of the deviation of critical boron concentration (value calculated by cosNU - value observed in site) is within $\pm 100 \mathrm{ppm}$. Critical boron concentration is the comprehensive index for measuring the core reactivity and it relates closely with the safety operation of the plant. The safety limit of it is $100 \mathrm{ppm}$. Therefore, if overrun during operation appears, deep analysis is necessary until a reasonable explanation is given. As shown in figure 1, the critical boron concentration of each of the burnup point meets the criteria of $100 \mathrm{ppm}$, with the biggest deviation of $63.1 \mathrm{ppm}$ in Unit 1 and $60.2 \mathrm{ppm}$ in Unit 2. And most of the deviations of Units 1 and 2 are within 50ppm and with one-way consistency. The deviations can be modified by reactivity for the consistency between the calculated and 
observed data which meets the site operation requirements.

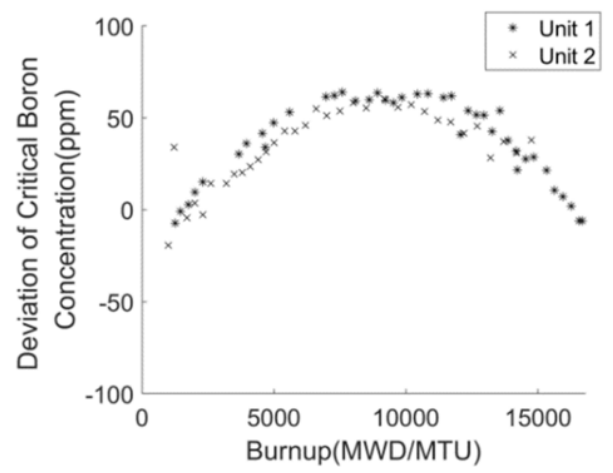

Figure 1. Deviation of critical boron concentration.

\subsection{Heat Pipe Factor of Nuclear Enthalpy Rise}

The deviation of heat pipe factor of nuclear enthalpy rises (value calculated by cosNU*1.04 - value observed in site, $4 \%$ is the uncertainty of the factor) between the observed value in site and the value calculated by cosNU is shown in figure 2 . The acceptance criteria of the deviation is within $\pm 3 \%$. The biggest deviation of Unit 1 is $2.14 \%$ and Unit 2 is $1.3 \%$. Most of the deviation of the two units are near $1 \%$ which meets the requirement of the acceptance criteria. At the same time, the calculated heat pipe factor of nuclear enthalpy rise of Unit 1 is up to 1.504 and the calculated heat flux hot spot factor of Unit 2 is up to 1.554. And as the burnup arises, the axial burnup distribution is more even. Together with the drop of the heat pipe factor of nuclear enthalpy rise, the field operation limit is 1.72 . This means enough operation margin to meet the requirements of field operation at any time.

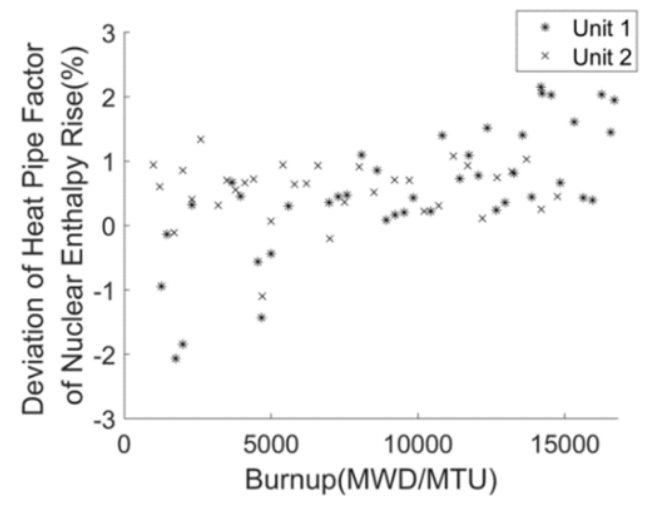

Figure 2. Deviation of nuclear enthalpy rise hot channel factor.

\subsection{Heat Flux Hot Spot Factor}

The deviation of heat flux hot spot factor ((value calculated by $\cos \mathrm{NU}^{*} 1.0815$ - value observed in site)/value observed in site, $8.15 \%$ is the uncertainty of the factor) between 
the observed value in site and the value calculated by cosNU is shown in figure 3 . The acceptance criterion of the deviation is within 5\%. The biggest deviation of Unit 1 is $-4.7 \%$ and Unit 2 is $-4.7 \%$. Most of the deviation of the two units are near $-3 \%$ which meets the requirement of the acceptance criteria. At the same time, the calculated factor of Unit 1 is up to 2.015 and Unit 2 up to 2.054. And as the burnup arises, the axial burnup distribution is more even. Together with the drop of the heat pipe factor of nuclear enthalpy rise, the field operation limit is 2.6. This means enough operation margin to meet the requirements of field operation at any time.

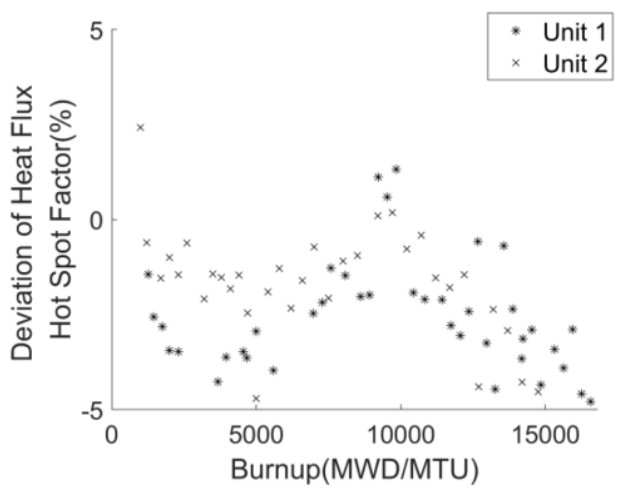

Figure 3. Deviation of heat flux hot channel factor.

\subsection{AO (Axial Offset)}

The deviation of the core AO (value calculated by cosNU-value observed in site) between the observed value in site and the value calculated by $\cos \mathrm{NU}$ is shown in figure 4 . The acceptance criteria of the deviation is within $5 \%$. The biggest deviation of Unit 1 is $3.5 \%$ and Unit 2 is $3.4 \%$ which both meet the requirement of the acceptance criteria. Most of the deviation of the two units are near $2 \%$ which meets the requirement of the acceptance criteria. As the burnup arises, the axial burnup distributions of the two units are more even and move toward positive direction until stability. The calculated AO by cosNU meets well with the requirement of control range between $[-5,+5]$ and the requirements of field operation.

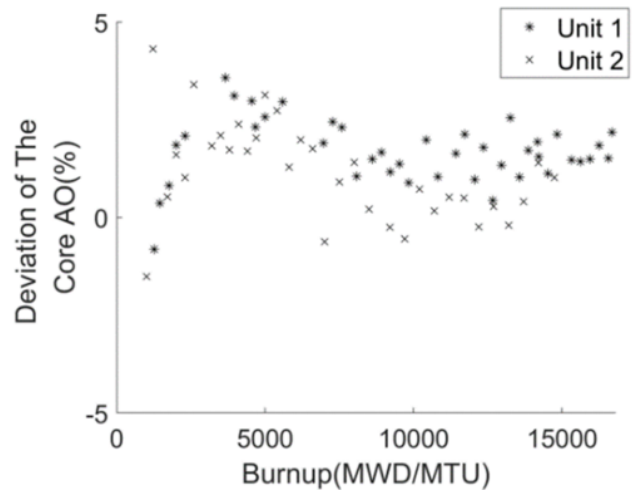

Figure 4. Deviation of heat axial offsets. 


\section{Conclusions}

Based on the data from the first cycle operation data of Units 1 and 2 in Haiyang Nuclear Power Plant, this paper proposed a study on the check calculation of COSINE software package for reactor core design, and then finished the verification and evaluation work of the software. The results from check calculation show that the values of the critical boron concentration, heat pipe factor of nuclear enthalpy rise, heat flux hot spot factor and AO calculated by cosNU at full power are in a good agreement with the values observed in operation site and all meet the technical requirements of the power plant. We believe that cosNU can calculate the operation limit parameters of the reactor core AP1000 in an accurate and reliable way on the basis of the above demonstration.

\section{References}

[1] Chen Y X, Liu Z Q, Hu X Y, et al. 2013 Core development and preliminary test verification of COSINE package core physical analysis program Atomic Energy Science and Technology 47 (Supplement) 365-368.

[2] Ge W, Yang Y H, Liu S, et al. 2016 Independent key design software for large advanced PWR nuclear power plant and development of COSINE software package 38 (7) 39-44.

[3] Su J C, Wang X, Yan Y H, et al. 2018 Fuel Consumption Calculation Model. User Manual of COSINE Software Component Parameter Calculation Program V1 (Beijing: Nuclear Power Software Technology Center of State Power Investment Research Institute Co., Ltd.) pp 19-22.

[4] Han W J, Su J C, Quan G P, et al. 2018 Engineering Automatic Sequence. User Manual of COSINE Software Core Physical Analysis Program V2.1 (Beijing: Nuclear Power Software Technology Center of State Power Investment Research Institute Co. Ltd.) pp 42-52.

[5] Hone M J 2016 The Nuclear Design and Core Management of the Haiyang Nuclear Power Plant Unit 1 Cycle 1.

[6] Bi G W, Tang C Y, Yang B, et al. 2017 Calculation method of neutron response of vanadium self-powered detector Nuclear Techniques $\mathbf{4 0} 060604$.

[7] Liu W Z, Li S C, Hu T X, et al. 2015 The introduction of self-powered detector and study for its escape probability calculation algorithm for $\beta$ particle Nuclear Electronics \& Detection Technology 35 5-7. 